\title{
Orthodontic outreach clinics - no marked advantages or disadvantages
}

\author{
For patients requiring orthodontic treatment are outreach clinics an effective \\ way of delivering services?
}

O'Brien K, Mattick R, Mandall N, Wright J, Conboy F, Gosden T. Are specialist outreach clinics for orthodontic consultation effective? A randomised controlled trial. Br Dent J 191:203-207, 2001

Design Single centre randomised controlled trial.

Intervention Allocation of referred patients to outreach or main base consultation appointments.

Outcome measure The outcome of consultation, the cost and duration of the visit and the carer/patient's perceptions of the visit.

Results There were no differences in outcome of the consultation. While consumer travel costs and the duration of appointments were significantly higher for the main base clinics, these differences were not great. However, consumers preferred to attend an appointment in an outreach clinic.

Conclusions There do not appear to be marked advantages or disadvantages in providing consultation appointments for orthodontics in outreach clinics.

\section{Commentary}

Over recent years a wide variety of specialist hospital services have developed outreach sessions in primary care settings. Outreach clinics are seen as a way of retaining some local service delivery in the face of increasing centralisation of specialist services. This approach is popular with patients or carers who after all are the ultimate 'customers'. The convenience of local access, reduced travel and contact in a familiar local setting are clear. Primary care clinicians appreciate the direct contact with specialists. Primary care commissioners believe it may reduce costs.

The Cochrane Library review on specialist outreach clinics ${ }^{1}$ looked at a range of medical services and the reviewers concluded specialist outreach can improve access, outcomes and service use. This well conducted trial on orthodontic outreach clinics is equally timely and relevant for those planning orthodontic services.

The study appears to show no major differences between the two models under investigation but the authors indicate that more work may be required to understand the different approaches. For example although cost effectiveness has been assessed, it has not been looked at in depth. The true opportunity costs need to be assessed for patients of the increased travel time taken for a visit to the main hospital base (mean $0.63 \mathrm{~h}$ ) compared with the satellite clinic (mean $0.37 \mathrm{~h}$ ) nor the opportunity cost of orthodontists being out of their hospital base. Interestingly the duration of the appointments was shorter at the outreach centre. The reasons for this also require further investigation. It could well be that the use of outreach may increase patient throughput which does not appear to have been addressed in the discussion.

It should also be noted that this study investigated one aspect of service delivery i.e. the 'triage' appointment. Few patients needed onward referral to the main base for specialised radiographs as the first appointment acted as a filter for secondary care. Long-term outcomes need to be assessed although the study suggested that providing consultation appointments in an outreach clinic did not influence the decisions of the orthodontists

This study is important in that it shows that there are no marked disadvantages from the provision of this form of outreach service and it helps to provide some clear evidence for those looking at this type of service. The approach fits in very well with the concept of integrated orthodontic services combining hospital and community based specialists.

However there are other important questions that need to be addressed before there is enough evidence to answer the wider question posed in our abstract. Moreover even when we have the evidence, there is still the major hurdle of getting it translated into evidence-based policy making.

\section{Alan Lawrence}

Chair, Evidence-based Dentistry Centre, Institute of Health

Sciences, Oxford

1. Gruen RL, Weeramanthri TS, Knight SE, Bailie RS. Specialist Outreach Clinics in Primary Care and Rural Hospital Settings (Cochrane Review), Issue 1. Chichester, UK: The Cochrane Library, 2004.

Evidence-Based Dentistry (2004) 5, 13.

doi:10.1038/sj.ebd. 6400240 